\title{
X-Ray Microscopy at Sirius, the New Brazilian Synchrotron Light Source
}

\author{
Harry Westfahl Jr. ${ }^{1 *}$, Hélio C. N. Tolentino ${ }^{1}$, Florian Meneau ${ }^{1}$, Nathaly Archilha ${ }^{1}$, Narcizo Marques de \\ Souza Neto ${ }^{1}$, Liu Lin ${ }^{1}$, Lucas Sanfelici ${ }^{1}$, Bernd Christian Meyer ${ }^{1}$, Jean Marie Polli ${ }^{1}$, Eduardo Miqueles ${ }^{1}$ \\ 1. Laboratório Nacional de Luz Síncrotron - LNLS/CNPEM, CP 6192, 13083-970 Campinas, SP, Brazil \\ * Corresponding author, westfahl@lnls.br
}

Since the early days of X-ray microscopy, new developments in light sources, X-ray optics, detection and data processing schemes have revolutionized our ability to study biological and synthetic materials in the micro and nanoscale, by means of multi-dimensional images with high elemental, chemical and magnetic sensitivity [1]. Particularly, in the last two decades, the enhancement in brightness and stability from second- to third-generation storage rings led to a dramatic increase in the flux of coherent X-rays that paved the way to scanning nanoprobe and lensless X-ray imaging schemes [2]. From the current third- to the coming fourth-generation storage rings a gain of the order of thousands of times in coherent flux is expected, allowing in a few seconds the acquisition of multi-dimensional images that nowadays may require several hours. This will be translated into faster and more user-friendly X-ray microscopes that can mass produce results and become available, either to specialists or to the novice, in an even broader range of scientific themes than the ones exploited today. Furthermore, these advances will likely evolve into novel ways of exploiting these high-speed gains, with a better integration between x-ray imaging and artificial intelligence, from data acquisition to data analysis.

Sirius, the new Brazilian Synchrotron Light Source, is the largest and most complex scientific infrastructure ever built in Brazil and one of the first $4^{\text {th }}$-generation Synchrotron Light Sources to start operation worldwide [3]. Its $518 \mathrm{~m}$ circumference electron storage ring is based on a 5BA lattice with a (bare machine) horizontal emittance of $250 \mathrm{pm}$.rad for a $3 \mathrm{GeV}$ beam. A further reduction to $150 \mathrm{pm} . \mathrm{rad}$ will be achieved with the extra damping provided by the installation of insertion devices. Currently, more than $85 \%$ of the building construction is completed, the installation of accelerator components is underway, and the installation of beamline parts will commence in the second semester of 2018. The design and construction principles of this new light source were envisaged to optimize production and use of coherent X-rays, with innovations ranging from the design of the multi-bend magnetic lattice and modified delta undulators to floor stability, high-dynamic active feedback opto-mechanical devices and fast, low noise area detectors. Starting from the design of the 5BA magnetic lattice, a large effort has been made to not only achieve a low emittance electron beam but also to improve its phase-space matching to the photon beam from undulators. Such matching is achieved when the beta functions in the middle of the straight sections reach $\beta_{\mathrm{x} / \mathrm{y}} \sim 1 \mathrm{~m}$. These optimizations resulted in a theoretical coherent flux gain of almost twice as much as suboptimal machine designs with the same emittance [4]. But, even with high coherent fluxes, it is paramount to maintain the beam stability from source to sample and detector, since noise from mechanical vibrations degrade coherence. A proper mechanical design of beamline components is thus required, with even higher stability performances. To tackle these challenges, we have applied fundamentally different dynamic architectures in the design of some of the most critical opto-mechanical devices of the Sirius beamlines (such as the DCM [5] and mirror mechanics), based on voice-coil actuators, leaf-spring mechanisms and laser interferometry feedback, in a high-bandwidth closed-loop control. All these systems finally require fast and reliable detection schemes, coupled with robust algorithms implemented in clusters of graphical processing units (GPU). Large area detectors based on gapless arrays of the Medipix $3 \mathrm{RX}$ chips are being constructed to enable 
the coherent imaging schemes that will be employed in the Sirius beamlines. Their electronic readout architecture design will sustain the inherent $\mathrm{kHz}$ frame rate of the Medipix ASIC, even when combined in larger panels of 36 to 144 chips (or 2.4 to 9.4 Mpixel, respectively). This readout systems will also be able to deliver high frame rate images through 100 to $200 \mathrm{Gbps}$ optical links to GPU servers, where algorithms of phase retrieval or tomographic computation will be executed in real time. The first installation phase will contemplate four beamlines based on x-ray undulators (for macromolecular crystallography, scanning x-ray nanoprobe, coherent x-ray scattering and extreme conditions), one beamline based on soft x-ray undulator (for ARPES and RIXS) and one beamline for cone beam highenergy X-ray tomography. The latter is based on the $3.2 \mathrm{~T}$ superbend present in the middle of each 5BA of the magnetic lattice. Therefore, 4 out of the 6 initial beamlines will exploit the low emittance properties of the $4^{\text {th }}$ generation storage ring for X-ray microscopy with nanometer resolution. Figure 1 shows an aerial view of Sirius building construction early March this year and Table 1 lists Sirius main parameters.

\begin{tabular}{ll}
\hline Parameter & Value \\
\hline Beam energy $(\mathrm{GeV})$ & 3.0 \\
Circumference $(\mathrm{m})$ & 518.4 \\
Lattice & $20 \times 5 \mathrm{BA}$ \\
Natural emittance (nm.rad) & $0.25^{*} \rightarrow 0.15$ \\
Minimum Horizontal/Vertical $\beta(\mathrm{m})$ & $1.5 / 1.5$ \\
Critical energy of central BM $(\mathrm{keV})$ & 19 \\
Nominal current $(\mathrm{mA})$ & 350 \\
Straight sections & $5 \times 7 \mathrm{~m} ; 15 \times 6 \mathrm{~m}$ \\
Energy spread $(\%)$ & 0.085 \\
\hline
\end{tabular}

Table 1 Sirius Main Parameters. *Bare machine

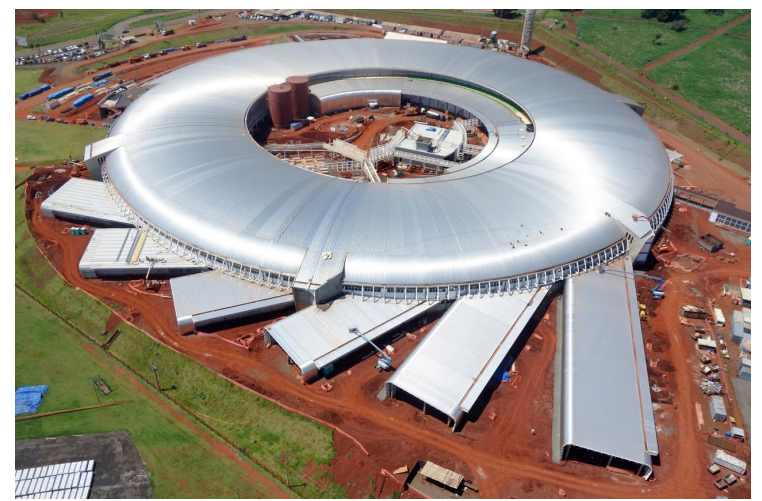

Figure 1 Aerial view of Sirius, March 2018. Six beamlines are $100 \mathrm{~m}-150 \mathrm{~m}$ long.

The Coherent X-Ray Nanoprobe Beamline (CARNAÚBA) [6] is a $150 \mathrm{~m}$ long, tender-to-hard X-ray beamline with two separated experimental stations, one for sub-micrometer resolution and another for nanometer resolution scanning probe, to cover several analysis techniques, including nXRD, nXAS, $\mathrm{nXRF}, \mathrm{nXEOL}$ and spectral ptychography with 2D and 3D imaging capabilities. The vertically polarized beam produced by a modified-Delta undulator allows for a completely side bounce optical layout from the source up to the end-stations, granting higher mechanical stability for all components. An in-house horizontal deflection four-bounce crystal monochromator $(4 \mathrm{CM})$ continuously covers the energy range of 2-15 keV with a resolution of $\Delta \mathrm{E} / \mathrm{E}=10^{-4}$. The achromatic CARNAÚBA focusing optics, based on state-of-the-art KB mirrors, delivers a coherent flux at $\operatorname{Si}(111)$ bandwidth, from $10^{11}$ to $10^{12}$ $\mathrm{ph} / \mathrm{s} / 100 \mathrm{~mA}$, with nearly diffraction limited focus, maintaining a relatively large working distance of about $50 \mathrm{~mm}$. In the nanoprobe end-station the round focus spot size is around $120 \mathrm{~nm}$ at $2 \mathrm{keV}$ and 30 nm above $8 \mathrm{keV}$, with $2 \mathrm{NA} \approx 5 \mathrm{mrad}$, reaching flux densities of above $10^{8} \mathrm{ph} / \mathrm{s} / \mathrm{nm}^{2}$. A high-dynamic actuation on the vertical and horizontal KB mirrors will deliver a fast-scanning capability in the 10 to $100 \mathrm{~Hz}$ range for fast fly-scan operation in spectral ptychography imaging.

The Coherent and Time Resolved X-Ray Scattering beamline (CATERETÊ) is optimized to perform Coherent X-ray Diffraction Imaging (CXDI) and X-ray Photon Correlation Spectroscopy (XPCS), covering a similar energy range and with the same undulator and monochromator of the CARNAÚBA beamline. Instead of a nanofocus, the CATERETE optical layout will be based on two side bounce 
mirrors, designed to deliver a nearly round, approximately $40 \mu \mathrm{m}$ diameter beam spot at the sample position, providing a plane-wave pencil beam within the waist of a few meters focal depth [7]. This layout delivers a coherent flux of up to $10^{13} \mathrm{ph} / \mathrm{s} / 100 \mathrm{~mA}$ at the $\mathrm{Si}(111)$ bandwidth. Associated with a 9.4 Megapixel Medipix 3RX detector array, a $30 \mathrm{~m}$ flight path, an air-bearing goniometry and a cryogenic sample cooling, X-ray microscopy in this beamline is expected to achieve a few tens of nanometer resolution in plane-wave 3D X-ray diffraction microscopy or ptychographic X-ray computed tomography with the addition of FZPs stabilized through a laser interferometric feedback system.

The EMA (Extreme conditions Methods of Analysis) beamline will be able to exploit different imaging modes for studying materials under extreme temperatures, pressures and magnetic fields, with a careful combination of sample environment and detection schemes. In particular, using double-diamond anvil cells (DDAC) this beamline will be able to analyse samples submitted to pressures of the order of several hundreds of GPa. Since the pressure built between the micro-anvils of the DDAC (in a region of about 10-20 microns) is likely very inhomogeneous, the scanning X-ray diffraction imaging with a 50 $\mathrm{nm}$ beam and a $1 \mathrm{mrad}$ divergence provided by the EMA beamline will be essential to map different states that may emerge within this micrometer region of extreme pressures. Another imaging mode available in the EMA beamline will be the direct tomography imaging by inelastic X-ray scattering from core electrons.

The energy range and field of views of the x-ray microscopes described in the last three beamlines are more compatible with micrometer to sub-millimeter samples. On Sirius, X-ray tomography for larger and denser samples will be available in the Micro and Nano X-Ray Tomography beamline (MOGNO) that will deliver a conical X-ray beam, diverging from a round $100 \mathrm{~nm}$ spot. This secondary source is created by demagnifying the micrometer source of the $3.2 \mathrm{~T}$ superbend using a multilayer coated KB mirror system with a $2 \%$ energy bandwidth. The two possible operation energies of MOGNO will be 27 $\mathrm{keV}$ and $68 \mathrm{keV}$, with total fluxes of $10^{12} \mathrm{ph} / \mathrm{s}$ and $10^{11} \mathrm{ph} / \mathrm{s}$, respectively. In both modes a 2.4 Megapixel Medipix 3RX detector array with high-Z sensors, placed at more than $10 \mathrm{~m}$ from the source will be fully illuminated by the sample projection image. By exploiting the variable field of view and pixel demagnification of the cone beam geometry, samples with sizes ranging from a few hundreds of microns to tens of millimeter can be imaged in phase contrast or absorption contrast tomography, with resolutions varying from a hundred nanometers to tens of microns, respectively.

In summary, we expect that the range of beamlines offered in Sirius will provide several flavors of x-ray imaging tools with unprecedented speeds and resolutions, that will bring new truths to our current understanding of the nanostructure of hierarchical, inhomogeneous composite systems [8].

[1] Kirz, J. \& Jacobsen, C., J. Phys. Conf. Ser. 186, (2009), 012001

[2] Chapman, H. N. \& Nugent, K. A., Nat. Photonics 4, (2010), 833

[3] Rodrigues, A. R. D. et al., Proc. 7th Int. Particle Accelerator Conf. (2016), 2811

[4] Liu, L. and Westfahl Jr., H., Proc. 8th Int. Particle Accelerator Conf. (2017), TUXA1.

[5] Geraldes, R.R. et al., Proceedings of MEDSI2016 (2016) TUCA05

[6] Tolentino, H. C. N. et al., Journal of Physics: Conference Series 849 (1),(2017) 012057

[7] Westfahl Jr., H. et al., Journal of synchrotron radiation 24 (3) (2017)

[8] The Sirius project is funded by the Brazilian Ministry of Science, Technology, Innovations and Communications. 
https://doi.org/10.1017/S1431927618013235 Published online by Cambridge University Press 